\title{
Innate recognition of microbial-derived signals in immunity and inflammation
}

\author{
Yue Zhang \& Chunli Liang* \\ Department of General Surgery, East Hospital, Tongji University School of Medicine, Shanghai 200120, China
}

Received October 12,2016; accepted October 27,2016; published online November 23, 2016

\begin{abstract}
Microbes generate a vast array of different types of conserved structural components called pathogen-associated molecular patterns (PAMPs), which can be recognized by cells of the innate immune system. This recognition of "nonself" signatures occurs through host pattern recognition receptors (PRRs), suggesting that microbial-derived signals are good targets for innate immunity to discriminate between self- and nonself. Such PAMP-PRR interactions trigger multiple but distinct downstream signaling cascades, subsequently leading to production of proinflammatory cytokines and interferons that tailor immune responses to particular microbes. Aberrant PRR signals have been associated with various inflammatory diseases and fine regulation of PRR signaling is essential for avoiding excessive inflammatory immune responses and maintaining immune homeostasis. In this review we summarize the ligands and signal transduction pathways of PRRs and highlight recent progress of the mechanisms involved in microbe-specific innate immune recognition during immune responses and inflammation, which may provide new targets for therapeutic intervention to the inflammatory disorders.
\end{abstract}

microbial-derived components, PRR signaling, immune regulation, innate immunity, inflammation

Citation: Zhang, Y., and Liang, C. (2016). Innate recognition of microbial-derived signals in immunity and inflammation 59, 1210-1217. Sci China Life Sci. doi: 10.1007/s11427-016-0325-6

\section{INTRODUCTION}

Hosts encounter different classes of microbes all the time and they must have proper mechanisms to sense invariant structural motifs such as lipids, proteins, glycans, lipoproteins or nucleic acids of invading pathogens including bacteria, fungi and viruses. Rapid innate immune responses to eliminate the infectious agents depend largely on the recognition to microbes and their products by a broad array of pattern recognition receptors (PRRs) (Iwasaki and Medzhitov, 2015). This complex receptor system consist of four main types of sensors: Toll-like receptors, RIG-like receptors which detect cytoplasmic viral RNAs, intracellular NOD-like receptors

\footnotetext{
*Corresponding author (email: liang2006718@sina.com)
}

and cytoplasmic sensor of DNA (Broz and Monack, 2013). In spite of having recognition of overlapped spectrum of pathogens, extensive research in the past decade has identified that the molecular bases of PRRs-ligand interactions and outcomes are quite different. Upon innate recognition of pathogens, multiple signaling pathways are initiated, culminating in induction of inflammatory mediators including inflammatory cytokines, chemokines, and type I IFNs, essential in host defense (Carpenter et al., 2014). Inflammation involving both innate and adaptive immunity is a normal response to infection, while excessive inflammatory responses can induce considerable tissue injury and can be highly detrimental to the host (Cao, 2016). A proper response to maintain host-microbial homeostasis and induce antimicrobial defense relies on specific positive or negative 
regulators to avoid a prolonged or exaggerated activation of inflammatory immune responses.

In this review, we will summarize the biological role of microbial components recognition by PRRs and focus on recent advances in the molecular mechanisms of the regulation of PRR-mediated immune response. Understanding the microbe-specific innate immune recognition will provide us with ways and means to develop therapeutic targets for prevention or treatment of inflammatory diseases.

\section{INNATE RECOGNITION OF MICROBIAL- DERIVED COMPONENTS BY PRRS}

Hosts are constantly exposed to the threat of microbial species including bacteria, fungi and viruses. Invading microbial organisms are the causative agents of various diseases and are characterised by specific arrangements of key molecules called pathogen associated molecular patterns (PAMPs) (Chu and Mazmanian, 2013). Well-known PAMPs mainly consist of the bacterial lipopolysaccharides (LPS), peptidoglycans, flagellin, fungal polysaccharides and viral nucleic acids (Qiao and $\mathrm{Wu}, 2015)$. These specific molecular patterns of components are recognized by several different families of PRRs on multiple cell types. Meanwhile, the same microbial-derived components may also be recognized by different PRRs.

\section{Innate immune pattern-recognition receptors}

Extensive work over the years has led to the discovery and characterization of multiple PRR families in different cellular compartments (Sellge and Kufer, 2015). So far, there are ten TLRs with distinct ligand-binding specificities in humans and twelve TLRs in mice. These type I transmembrane proteins are present on the plasma membrane (TLR1, TLR2, TLR4, TLR5, TLR6 and TLR11) or within the endosomal and lysosomal compartment (TLR3, TLR7, TLR8, TLR9 and TLR10) (Gay et al., 2014). The extracellular domain containing varying leucine-rich-repeat (LRR) motifs is involved in the recognition of PAMPs, while the intracellular TIR (Toll/IL-1R) domain activates signaling pathways. As cytosolic PRRs, three members of the RLR family have been identified: RIG-I (retinoic acid-inducible gene I), MDA5 (melanoma differentiation associated factor 5) and LGP2 (laboratory of genetics and physiology 2). RIG-I and MDA5 share a C-terminal domain (CTD) involved in RNA recognition, a central DEAD box helicase/ATPase domain and two N-terminal caspase activation and recruitment domains (CARDs) required for downstream signaling. LGP2, however, lacks the N-terminal CARD domains and therefore cannot induce downstream signaling. In addition to the already mentioned PRRs, a group called NLRs are also expressed intracellularly and consist of 23 members in humans versus 34 members in mice. NLR proteins have a common domain architecture that includes a $\mathrm{C}$-terminal
LRR domain, a central nucleotide-binding oligomerization domain (NOD), and an N-terminal effector domain. They can be divided into five subfamilies: NODs, NACHT-, LRR-and pyrin-domain (PYD)-containing protein (NLRPs), neuronal apoptosis inhibitory protein (NAIPs), CIITA and ICE-protease-activating factor (IPAF). During infections with intracellular DNA-containing microbes, DNA may be released from the microbe and end up in the cytosol to allow DNA sensing. Significant progress has been made in the last few years relating to cytosolic DNA sensing, and more than ten cytosolic receptors of DNA have been proposed (Barrat et al., 2016).

\section{Innate recognition of bacterial-derived components}

Upon interaction with bacteria, host activates innate immune system following the detection of a variety of PAMPs which are unique to bacteria. Different works highlight the relevance of the PRR-mediated responses in host defense against bacteria (Thaiss et al., 2016). TLR1/TLR2 or TLR6/TLR2 complex forms a pocket for its ligand, triacylated lipoprotein and diacylated lipoprotein, respectively. Together with myeloid differentiation factor 2 (MD2), TLR4 can recognized bacterial cell-wall component such as LPS. It has been revealed that TLR5 can recognize flagellin of several mobile bacterial species (Xiao et al., 2015). NOD1 and NOD2 are NLRs that recognize the structures of bacterial peptidoglycans, $\gamma$-D-glutamyl-mesodiaminopimelic acid (iE-DAP) and muramyl dipeptide (MDP) derived from the bacterial cell wall. Other NLRs, such as IPAF, NALP3, NLRP12, NALP1 and NLRP1 are known to detect perotoxins, ATP, anthrax lethal toxin and type 3 secretion system (T3SS) from invading bacteria (Zhao and Shao, 2016). Although DNA with a CpG motif is sensed in endosomes by TLR9, several studies have indicated the existence of cytosolic DNA PRRs in the recognition of infections with intracellular bacteria such as AIM2 and DAI. TLR7 recognizes bacterial RNA in some cell types and bacterial RNA:DNA hybrids are a unique class of microbe-associated molecular pattern for NLRP3 (Kailasan Vanaja et al., 2014). Pyrin has recently been reported to sense the pathogenic bacteria-induced modification of Rho GTPases, which helps to distinguish pathogenic bacteria from non-pathogenic ones (Yang et al., 2014).

\section{Innate recognition of viral-derived components}

Virus infections can have devastating outcomes for the host and the resistance of immunity must therefore be mediated by innate recognition immune receptors that bind viral components and inhibit viral replication (Chan and Gack, 2016). Parallel to TLR2 and TLR4 to recognize viral structural protein, it has been revealed that TLR3 detects viral double-stranded (ds) RNA in the endolysosome, while mouse TLR7 and human TLR7/8 recognize single-stranded (ss) RNAs from RNA viruses. Studies in RLR-deficient 
mice and cells have demonstrated essential and differential requirements for RIG-I and MDA5 to viral RNA. RIG-I has been identified to bind with high affinity to an RNA with short blunt dsRNA bearing a 59 triphosphate $(<50 \mathrm{bp})$ and short poly(I:C) $(<300 \mathrm{bp})$, which provides a mechanism for virus self-discrimination by RIG-I. Other RIG-I ligands lacking a 5'-ppp such as small structured RNaseL cleavage-derived RNAs, short ( $25 \mathrm{bp}$ ) and long (>200 bp) dsRNA have also been found (Yoneyama et al., 2015). Interestingly, RIG-I has also been found to mediate antiviral responses to RNAs bearing $5^{\prime}$-diphosphates (5'-pp) (Goubau et al., 2014). In contrast, MDA5 recognizes long poly(I:C) and long dsRNA (1-2 kb), which is produced during replication of sense-strand ssRNA viruses. Additional DExD/H-box helicases including DDX3, DHX9, DDX60 and DHX36 have also been recently reported to act as cytosolic sensors of viral nucleic acids (Fullam and Schröder, 2013). Moreover, interferon-induced proteins with tetratricopeptide repeats (IFITs) are a family of proteins in the preferential binding to mis- or un-modified RNA and the viral replication proteins in the cytoplasm (Vladimer et al., 2014). More recently, DAI (DNA-dependent activator of IRFs), AIM2 (absent in melanoma 2), Pol III (DNA-dependent RNA polymerase III), the IFN inducible protein IFI16, cyclic GMP-AMP synthase (cGAS), Ku70, Sox2, DNA-PK, LRRFIP1 and MRE11 have been implicated to detect cytoplasmic DNA recognition is important for the production of type I IFNs to infection with DNA viruses (Dempsey and Bowie, 2015; Xia et al., 2015).

\section{Innate recognition of fungal-derived components}

Collaboration between different classes of PRRs is important for fungal recognition which is mediated by TLR2/6, TLR4, TLR7 and TLR9 sensing phospholipomannan, O-linked mannan, RNA and phagocytosed fungal DNA, respectively (Netea et al., 2015). NALP3 has also been identified as the critical NLR family member that transduces the fungal recognition signal to the inflammasome activation and pro-IL-1 $\beta$ processing (Feriotti et al., 2015). C-type lectin receptor Dectin-3 forms a heterodimeric PRR with Dectin-2 to bound $\alpha$-mannans more effectively, leading to potent inflammatory responses against fungal infections (Zhu et al., 2013). Recently, SHP-2 (Deng et al., 2015) has been identified to mediate C-type lectin receptor-induced activation of the kinase Syk and anti-fungal responses.

\section{REGULATION OF TLR SIGNALING PATHWAYS}

Recognition of microbial-derived components by TLRs except TLR3 transmit their signal with TIR domain through adaptor proteins Mal and MyD88 which then interact with IRAKs and TRAF6. Acting as an E3 ubiquitin protein ligase, TRAF6 activates a complex of TAK1, TAB1, and TAB2/3 leading to the phosphorylation of NEMO and the activation of an IKK complex (Bryant et al., 2015). This in turn activates MAP kinase cascades and NF- $\mathrm{KB}$ thus inducing production of inflammatory cytokines. Both TLR3 and TLR4 recruit the TIR adapter TRAM, and then use TRIF to recruit another signaling complex composed of TRAF3/TBK1/IKK- $i$ to activate IRF3 for the transcription of type I IFNs (Qian and Cao, 2013).

A coordinated balance between the positive and negative regulation of TLR signal-triggered innate inflammatory responses is required to ensure the most favorable consequence for the host to eradicate pathogens. To date, the modulation of TLR signaling pathways at multiple levels have been the subject of intensive research (Leifer and Medvedev, 2016). As membrane-associated regulator, constitutively expressed membrane MHC I can interacting with Fps which induces SHP-2-mdiated inhibition of TRAF6 and TBK1 (Xu et al., 2012) which lead to negatively regulation of TLR-triggered inflammatory responses. In addition, VEGFR-3 (Zhang et al., 2014c) can restrain TLR4-NF- $\mathrm{kB}$ activation and significantly attenuated proinflammatory cytokine production. MHC-I (Nair-Gupta et al., 2014) recruited from Rab11a selectively accumulate within phagosomes carrying microbial components, which engage TLR signaling. Several intracellular regulators such as Ctcf (Nikolic et al., 2014), RasGRP3 (Tang et al., 2014), PtpA (Wang et al., 2015), ZBTB20 (Liu et al., 2013b), Fbxo3 (Chen et al., 2013a), PP2A (Long et al., 2014) and glycolysis (Everts et al., 2014) operate as distinct checkpoints for fine-tuning TLR signal. Epigenetic alterations reprogram distinct functional sets of genes to both activate and repress transcription of hundreds of genes. In the nucleus, epigenetic alterations reprogram distinct functional sets of genes to both activate and repress the microbe-induced inflammation. Zhang et al. have recently reported that DNA modifier Tet2 is required to resolve inflammation by recruiting Hdac2 to selectively repress transcription of IL-6 via histone deacetylation (Zhang et al., 2015). Histone lysine methyltransferase Ezh1 has been identified to promote TLR-triggered inflammatory cytokine production by suppressing the TLR negative regulator Tollip (Liu et al., 2015). The transcription factor DREAM can transcriptionally repress A20 and thereby impaire the NF- $\mathrm{KB}$ signaling (Tiruppathi et al., 2014). As a multifunctional nuclear protein, death domain-associated protein 6 (Daxx) selectively represses IL-6 transcription through histone deacetylase 1 (HDAC1)-mediated histone deacetylation in TLR-induced macrophages (Yao et al., 2014). Kinases Mst1 and Mst2 has been identified to activate the GTPase Rac to promote TLR-triggered assembly of the TRAF6-ECSIT complex that is required for the recruitment of mitochondria to phagosomes and bactericidal activity (Geng et al., 2015), while Mst4 limits inflammatory responses through direct phosphorylation of the adaptor TRAF6 (Jiao et al., 2015). 
MicroRNAs are also emerging as important regulators in the regulation of TLR pathways (Zhang and Li, 2013).

\section{REGULATION OF RLR SIGNALING PATHWAYS}

At resting state, RIG-I adopts a rigid autoinhibited state where the CARD domains are sterically unavailable for signal transduction. Upon different RNA viruses infection, activated RIG-I and MDA5 interact with the mitochondrial adaptor MAVS and induces MAVS activation and oligomerization into a prion-like aggregate. The engagement of MAVS by RLRs causes a conformational change and thereby results in activating the downstream signaling cascades such as TBK1 and IKK kinases which lead to the expression of type I IFN and proinflammatory cytokines via NF- $\mathrm{B}$, IRF-3, and IRF-7 (Junt and Barchet, 2015).

Precise control of RLR signaling is critical for efficient viral clearance without harmful immunopathology, and several regulators of RLR signaling pathway have been reported. For example, DNA methyltransferase Dnmt3a selectively upregulates the production of type I interferons by maintaining high expression of HDAC9 to deacetylate TBK1 for activation of antiviral innate immunity (Li et al., 2016). RNA viruses can specifically upregulate the expression of Siglec-G (Chen et al., 2013b), Siglec1 (Zheng et al., 2015) and DAPK1 (Zhang et al., 2014a) which regulate antiviral innate immune response. Cytoplasmic STAT4 has been described to interact with E3 ligase CHIP and block RIG-I and CHIP association, resulting in preventing CHIP-mediated proteasomal degradation of RIG-I (Zhao et al., 2016). E3 ubiquitin ligase RING finger protein 122 (RNF122) can supress RIG-I-triggered antiviral innate response by targeting CARDs of RIG-I and mediating proteasomal degradation of RIG-I (Wang et al., 2016). A bicistronic mRNA of MAVS has been identified to encode a second protein miniMAVS to interfere with interferon production induced by full-length MAVS (Brubaker et al., 2014). Human OASL contributes to host antiviral responses by specifically enhancing RIG-I activation through its C-terminal ubiquitin-like domain (Zhu et al., 2014).

\section{REGULATION OF NLR SIGNALING PATHWAYS}

NLRs represent a large family of intracellular sensors that can detect pathogens and stress signals such as degradation products of peptidoglycans, microbial products, noninfectious crystal particles and ultraviolet irradiation (Wen et al., 2013). It has long been known that the NOD1 and NOD2 can interact with an adaptor protein RIP2 which mediate the activation of various transcription factors such as NF- $\kappa \mathrm{B}, \mathrm{MAPK}$, and IRFs to induce the production of proinflammatory cytokines, type I IFNs and antimicrobial peptides. A major function of another subfamily of NLRs such as NLRP1, NLRP3, NLRP6 (Wlodarska et al., 2014), and NLRC4 is in inflammasome activation. Inflammasome assembly has been described that recruit the common adaptor protein ASC to activate caspase-1, leading to the maturation of IL-1 $\beta$ and IL-18 maturation as well as pyroptosis. Interestingly, RNA viruses can also promote activation of the NLRP3 inflammasome through a RIP1-RIP3-DRP1 (Wang et al., 2014) signaling pathway.

The NLR activation has been implicated in the pathogenesis of several acquired inflammatory diseases (Dorfleutner et al., 2015). As an NLRP3-binding protein, a member of the family of mammalian NIMA-related kinases (Neks) Nek7 acts downstream of potassium efflux to regulate NLRP3 oligomerization and activation (Groß et al., 2016). Leucine-rich repeat Fli-I-interacting protein 2 (LRRFIP2) negatively regulates NLRP3 inflammasome activation by recruiting the caspase-1 inhibitor Flightless-I (Jin et al., 2013). The recent finding that dopamine D1 receptor (DRD1) signaling cause negative regulation of NLRP3 inflammasome via cAMP, which binds to NLRP3 and promotes its ubiquitination and degradation via MARCH7 (Yan et al., 2015). Endogenous COPs and POPs bind to caspase-1 and ASC respectively, resulting in sequestering them from the inflammasome complex (Krishnaswamy et al., 2014). Some microbial proteins, Omega-3, A20 and autophagy can also inhibit NLRP3 inflammasome activation (Yan et al., 2013; Vande Walle et al., 2014). More recently, prion-like polymerization of underlies signal transduction of NLRP3 and ASC in antiviral immune defense and inflammasome activation (Cai et al., 2014; Franklin et al., 2014) .

\section{REGULATION OF CYTOSOLIC DNA SENSORS DEPENDENT SIGNALING PATHWAYS}

One area of recent intense research has been the search for cytosolic DNA sensors dependent signaling pathways (Roers et al., 2016). DAI is able to bind right-handed spiral B-DNA and interact with TBK1, leading to activation of type I IFN responses. In addition, AIM 2 has been shown to activate caspase-1 via the inflammasome component ASC in response to cytosolic DNA. As the enzyme responsible for synthesizing 5'-ppp RNA from the poly(dA-dT) template, Pol-III detects cytosolic DNA and induces type I IFN through the RIG-I pathway (Paludan and Bowie, 2013). The STING pathway has now been shown to be the hub adaptor of the recently identified DNA sensors for activating innate immune gene transcription in response to infection by DNA pathogens (Barber, 2015). Recent studies have also revealed a key role in STING activation for cGAS that generates the cyclic dinucleotide (CDN), cyclic GMP-AMP (cGAMP) from GTP and ATP in a DNA-dependent manner (Chen et al., 2016). In addition, pan-viral specificity of IFN-induced genes reveals 
new roles for cGAS in antiviral pathways spanning several virus families (Schoggins et al., 2014). More recently, sequence-specific activation of cGAS by DNA structures containing unpaired guanosines flanking short (12- to 20-bp) dsDNA (Y-form DNA) structures has been found in primary HIV-1 cDNA (Herzner et al., 2015).

During DNA-based microbes invasion, negative regulators are required to prevent overwhelming immune responses and maintain homeostasis. For example, IRF1 (Man et al., 2015) and guanylate-binding proteins (Meunier et al., 2015) are required for activation of the AIM2 inflammasome during Francisella infection, while the PYRIN domain-only protein POP3 competes with ASC for recruitment to AIM2-like receptors and inhibits DNA virus-induced activation of inflammasomes (Khare et al., 2014). As a positive regulator, inactive rhomboid protein 2 (iRhom2) can mediate trafficking and stability of the adaptor STING to promote DNA-virus-triggered induction of type I IFN (Luo et al., 2016). Sumoylation by Trim 38 and desumoylation by Senp 2 regulate the stability of the DNA sensor cGAS to regulate the kinetics of response to DNA virus (Hu et al., 2016). NLRC3 was recently found to block the cytosolic DNA detection system to fine tune host response to intracellular DNA, DNA virus, and c-di-GMP (Zhang et al., 2014b). Also, the IFN-inducible E3 ligase TRIM21 has been found to inhibit cytosolic DNA responses by mediating DDX41 degradation, while unanchored K48-linked polyubiquitin synthesized by TRIM6 stimulates the interferon-IKK $\varepsilon$ kinase-mediated antiviral response (Zhang et al., 2013; Rajsbaum et al., 2014).

\section{ABERRANT SIGNALS OF INNATE RECOGNITION IN INFLAMMATORY DISEASES}

The importance of innate recognition of microbial-derived signals is underscored by aberrant PRR signals in innate immunity and the pathophysiology of various inflammatory diseases, such as endotoxin shock, inflammatory bowel disease (IBD), and rheumatoid arthritis (RA) (Qian et al., 2014). Therefore, identification and characterization of regulators of PRR signals will add mechanistic insights that underlie development of inflammatory diseases and may suggest possible drug targets for intervention of these diseases (Yuan and $\mathrm{Li}, 2016$ ). Accumulating studies have suggested that lack of positive adaptors of PRRs protects host from microbial pathogen challenge, while negative regulators deficiency can cause excessive inflammatory immune responses and increased susceptibility to endotoxic shock after microbial components stimulation (Liu et al., 2016). Recently, it has shown that Rhbdd3 plays a critical role in attenuating TLR3-triggered acute inflammation by controlling NK cell activation in liver, and suppresses the production of IL-6 in dendritic cells via K27-linked ubiquitination of the regulator
NEMO and was involved in the control of IBD (Liu et al., 2013a; Liu et al., 2014). The autophagy protein ATG16L1 has been identified to suppress Nod-driven inflammatory responses and contribute to the chronic inflammation associated with Crohn's disease (Sorbara et al., 2013). Moreover, Ash11, a H3K4 methyltransferase, was reported to enhance A20 expression and suppress subsequent IL-6 production in TLR-triggered macrophages, protecting mice from $E$. coli-inducedsepsis and collagen-II induced arthritis (Xia et al., 2013). More recently, it has been shown that LPS primes the proinflammatory innate immune response via TLR4 and can be considered a major hidden risk factor for osteoarthritis (Huang and Kraus, 2016).

\section{CONCLUSIONS}

Inflammation is a protective response by the body to harmful stimuli, such as microbial infection, tissue injury, and cardiac infarction. The function of inflammation is to ensure removal of detrimental stimuli, as well as a healing process for repairing damaged tissue, and return to homeostasis. Nevertheless, inflammation can also be detrimental and is linked to various severe diseases when activated chronically or in excess (Cao, 2016). For instance, it is well established that bacterial infection via TLR-mediated signal pathways is a major cause of sepsis and septic shock. Similarly, RLRs and new DNA receptors are associated with the process of host defense against microbial infection as well as development of some autoimmune diseases such as systemic lupus erythematosus. In addition, inflammasomes nucleated by NLRs integrate signals from metabolic and commensal systems contributing to inflammatory bowel diseases, allergy, metabolic dysfunction and type 2 diabetes.

With a dynamic and flexible regulation system comprising post-translational modifications, epigenetic modifications, metabolic modifications and microenvironment effect, PRR dependent innate signaling manages to prevent inappropriate activation or overactivation which might cause damaging inflammation to the host. In recent years, the field of microbial recognition has progressed tremendously in understanding the balanced control of innate immune responses (Qian et al., 2014). These new insights should pave the way for the development of new preventive or antimicrobial therapeutics such as improved vaccine adjuvants and vaccine strategies to target more optimally for preventing specific microbes (Figure 1). Such developments will be supported by continuing research aimed at the nature of microbial components that confer PRR recognition and specifically defining PAMP-PRR interactions. Further characterization of the regulators by which innate immune recognition signaling pathways are controlled should allow us to define new targets for drug treatment aimed at preventing and managing more effectively some autoimmune and inflammatory diseases. 


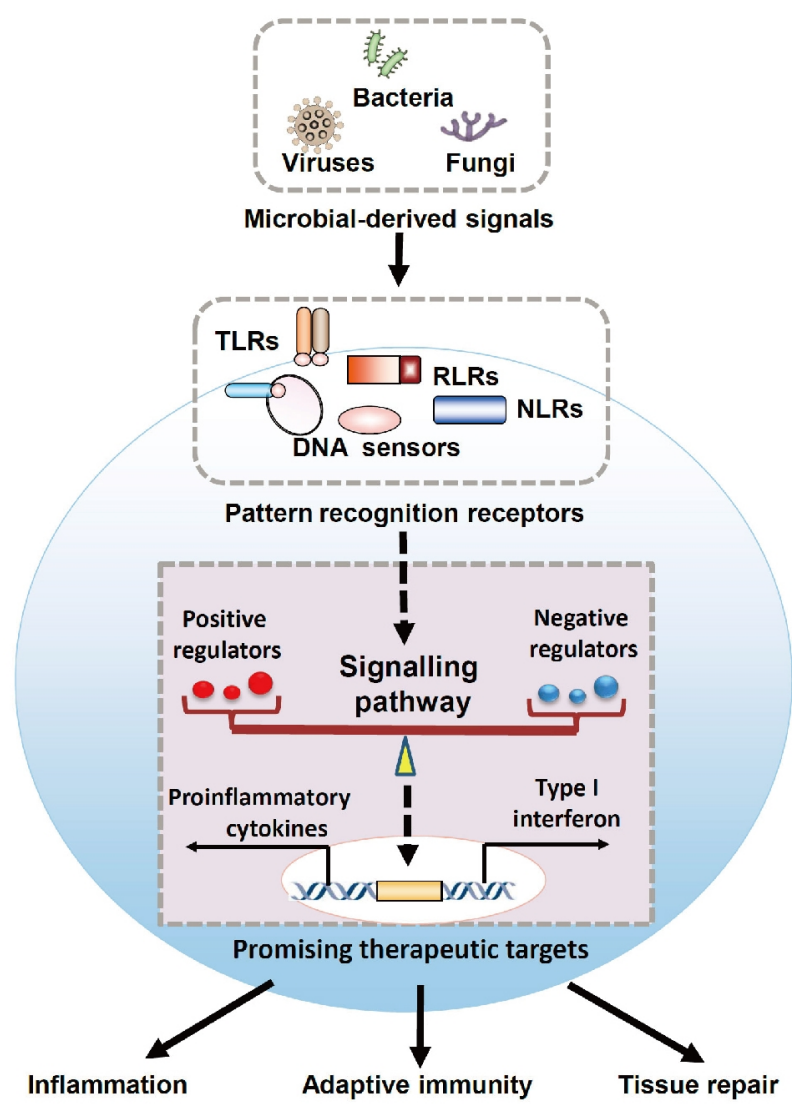

Figure1 (Color online) Promising therapeutic targets of innate recognition in inflammation. PRRs such as TLRs, RLRs, NLRs and cytoplasmic DNA sensors act as central integrators of a wide variety of signals, responding to diverse agonists mainly from invading bacteria, viruses and fungus. These microbial-derived signals participate in elaborate signaling pathways in multiple innate immune cells, and in turn induce transcription of genes leading to inflammation, tissue repair, and the initiation of adaptive immunity. The key to optimum health is immune balance, and this balance might be disturbed during diseases. Thus, the immune system must be tightly regulated by positive and negative regulators to maintain a balance of immune homeostasis. This new knowledge suggests that the targets to manipulate PRR pathways has great therapeutic potential for inflammatory immune diseases.

So far, several intriguing and important questions of the transduction and regulation of PRR-triggered innate signaling remain unresolved and new questions have arisen as a result of recent progress, which therefore present challenges for future research. Future studies will probably reveal additional mechanisms of innate immune recognition that how different PRRs activated by multiple PAMPs crosstalk to induce effective innate immune responses, and how do metabolic pathways, epigenetic modifiers, ion signaling, autophagy- and apoptosis-related molecules regulate or direct the responses of innate immune cells upon PRR stimulation.

Compliance and ethics The author(s) declare that they have no conflict of interest.

Barber, G.N. (2015). STING: infection, inflammation and cancer. Nat Rev Immunol 15, 760-770.

Barrat, F.J., Elkon, K.B., and Fitzgerald, K.A. (2016). Importance of nucleic acid recognition in inflammation and autoimmunity. Annu Rev Med 67, 323-336.

Broz, P., and Monack, D.M. (2013). Newly described pattern recognition receptors team up against intracellular pathogens. Nat Rev Immunol 13, 551-565.

Brubaker, S.W., Gauthier, A.E., Mills, E.W., Ingolia, N.T., and Kagan, J.C. (2014). A bicistronic MAVS transcript highlights a class of truncated variants in antiviral immunity. Cell 156, 800-811.

Bryant, C.E., Symmons, M., and Gay, N.J. (2015). Toll-like receptor signalling through macromolecular protein complexes. Mol Immunol 63, $162-165$.

Cai, X., Chen, J., Xu, H., Liu, S., Jiang, Q.X., Halfmann, R., and Chen, Z.J. (2014). Prion-like polymerization underlies signal transduction in antiviral immune defense and inflammasome activation. Cell 156, 1207-1222.

Cao, X. (2016). Self-regulation and cross-regulation of pattern-recognition receptor signalling in health and disease. Nat Rev Immunol 16, 35-50.

Carpenter, S., Ricci, E.P., Mercier, B.C., Moore, M.J., and Fitzgerald, K.A. (2014). Post-transcriptional regulation of gene expression in innate immunity. Nat Rev Immunol 14, 361-376.

Chan, Y.K., and Gack, M.U. (2016). Viral evasion of intracellular DNA and RNA sensing. Nat Rev Micro 14, 360-373.

Chen, B.B., Coon, T.A., Glasser, J.R., McVerry, B.J., Zhao, J., Zhao, Y., Zou, C., Ellis, B., Sciurba, F.C., Zhang, Y., and Mallampalli, R.K. (2013a). A combinatorial $\mathrm{F}$ box protein directed pathway controls TRAF adaptor stability to regulate inflammation. Nat Immunol 14, 470-479.

Chen, Q., Sun, L., and Chen, Z.J. (2016). Regulation and function of the cGAS-STING pathway of cytosolic DNA sensing. Nat Immunol 17, 1142-1149.

Chen, W., Han, C., Xie, B., Hu, X., Yu, Q., Shi, L., Wang, Q., Li, D., Wang, J., Zheng, P., Liu, Y., and Cao, X. (2013b). Induction of siglec-G by RNA viruses inhibits the innate immune response by promoting RIG-I degradation. Cell 152, 467-478.

Chu, H., and Mazmanian, S.K. (2013). Innate immune recognition of the microbiota promotes host-microbial symbiosis. Nat Immunol 14, 668-675.

Dempsey, A., and Bowie, A.G. (2015). Innate immune recognition of DNA: a recent history. Virology 479-480, 146-152.

Deng, Z., Ma, S., Zhou, H., Zang, A., Fang, Y., Li, T., Shi, H., Liu, M., Du, M., Taylor, P.R., Zhu, H.H., Chen, J., Meng, G., Li, F., Chen, C., Zhang, Y., Jia, X.M., Lin, X., Zhang, X., Pearlman, E., Li, X., Feng, G.S., and Xiao, H. (2015). Tyrosine phosphatase SHP-2 mediates C-type lectin receptor-induced activation of the kinase Syk and anti-fungal TH17 responses. Nat Immunol 16, 642-652.

Dorfleutner, A., Chu, L., and Stehlik, C. (2015). Inhibiting the inflammasome: one domain at a time. Immunol Rev 265, 205-216.

Everts, B., Amiel, E., Huang, S.C.C., Smith, A.M., Chang, C.H., Lam, W.Y., Redmann, V., Freitas, T.C., Blagih, J., van der Windt, G.J.W., Artyomov, M.N., Jones, R.G., Pearce, E.L., and Pearce, E.J. (2014). TLR-driven early glycolytic reprogramming via the kinases TBK1-IKK $\varepsilon$ supports the anabolic demands of dendritic cell activation. Nat Immunol 15, 323-332.

Feriotti, C., Bazan, S.B., Loures, F.V., Araújo, E.F., Costa, T.A., and Calich, V.L.G. (2015). Expression of dectin-1 and enhanced activation of NALP3 inflammasome are associated with resistance to paracoccidioidomycosis. Front Microbiol 6, 913.

Franklin, B.S., Bossaller, L., De Nardo, D., Ratter, J.M., Stutz, A., Engels, G., Brenker, C., Nordhoff, M., Mirandola, S.R., Al-Amoudi, A., Mangan, M.S., Zimmer, S., Monks, B.G., Fricke, M., Schmidt, R.E., Espevik, T., Jones, B., Jarnicki, A.G., Hansbro, P.M., Busto, P., Marshak-Rothstein, A., Hornemann, S., Aguzzi, A., Kastenmüller, W., and Latz, E. (2014). The adaptor ASC has extracellular and "prionoid" activities that propagate inflammation. Nat Immunol 15, 727-737.

Fullam, A., and Schröder, M. (2013). DExD/H-box RNA helicases as mediators of anti-viral innate immunity and essential host factors for viral replication. Biochim Biophys Acta 1829, 854-865.

Gay, N.J., Symmons, M.F., Gangloff, M., and Bryant, C.E. (2014). Assembly and localization of Toll-like receptor signalling complexes. Nat Rev Immunol 14, 546-558. 
Geng, J., Sun, X., Wang, P., Zhang, S., Wang, X., Wu, H., Hong, L., Xie, C., Li, X., Zhao, H., Liu, Q., Jiang, M., Chen, Q., Zhang, J., Li, Y., Song, S., Wang, H.R., Zhou, R., Johnson, R.L., Chien, K.Y., Lin, S.C., Han, J., Avruch, J., Chen, L., and Zhou, D. (2015). Kinases Mst1 and Mst2 positively regulate phagocytic induction of reactive oxygen species and bactericidal activity. Nat Immunol 16, 1142-1152.

Goubau, D., Schlee, M., Deddouche, S., Pruijssers, A.J., Zillinger, T., Goldeck, M., Schuberth, C., Van der Veen, A.G., Fujimura, T., Rehwinkel, J., Iskarpatyoti, J.A., Barchet, W., Ludwig, J., Dermody, T.S., Hartmann, G., and Reis e Sousa, C. (2014). Antiviral immunity via RIG-I-mediated recognition of RNA bearing 5'-diphosphates. Nature 514, 372-375.

Groß, C.J., Mishra, R., Schneider, K.S., Médard, G., Wettmarshausen, J., Dittlein, D.C., Shi, H., Gorka, O., Koenig, P.A., Fromm, S., Magnani, G., Ćiković, T., Hartjes, L., Smollich, J., Robertson, A.A.B., Cooper, M.A., Schmidt-Supprian, M., Schuster, M., Schroder, K., Broz, P., Traidl-Hoffmann, C., Beutler, B., Kuster, B., Ruland, J., Schneider, S., Perocchi, F., and Groß, O. (2016). $\mathrm{K}^{+}$efflux-independent NLRP3 inflammasome activation by small molecules targeting mitochondria. Immunity $45,761-773$.

Herzner, A.M., Hagmann, C.A., Goldeck, M., Wolter, S., Kübler, K., Wittmann, S., Gramberg, T., Andreeva, L., Hopfner, K.P., Mertens, C., Zillinger, T., Jin, T., Xiao, T.S., Bartok, E., Coch, C., Ackermann, D., Hornung, V., Ludwig, J., Barchet, W., Hartmann, G., and Schlee, M. (2015). Sequence-specific activation of the DNA sensor cGAS by Y-form DNA structures as found in primary HIV-1 cDNA. Nat Immunol $16,1025-1033$

Hu, M.M., Yang, Q., Xie, X.Q., Liao, C.Y., Lin, H., Liu, T.T., Yin, L., and Shu, H.B. (2016). Sumoylation promotes the stability of the DNA sensor cGAS and the adaptor STING to regulate the kinetics of response to DNA virus. Immunity 45, 555-569.

Huang, Z., and Kraus, V.B. (2016). Does lipopolysaccharide-mediated inflammation have a role in OA? Nat Rev Rheumatol 12, 123-129.

Iwasaki, A., and Medzhitov, R. (2015). Control of adaptive immunity by the innate immune system. Nat Immunol 16, 343-353.

Jiao, S., Zhang, Z., Li, C., Huang, M., Shi, Z., Wang, Y., Song, X., Liu, H., Li, C., Chen, M., Wang, W., Zhao, Y., Jiang, Z., Wang, H., Wong, C.C.L., Wang, C., and Zhou, Z. (2015). The kinase MST4 limits inflammatory responses through direct phosphorylation of the adaptor TRAF6. Nat Immunol 16, 246-257.

Jin, J., Yu, Q., Han, C., Hu, X., Xu, S., Wang, Q., Wang, J., Li, N., and Cao, X. (2013). LRRFIP2 negatively regulates NLRP3 inflammasome activation in macrophages by promoting Flightless-I-mediated caspase-1 inhibition. Nat Commun 4, 2075.

Junt, T., and Barchet, W. (2015). Translating nucleic acid-sensing pathways into therapies. Nat Rev Immunol 15, 529-544.

Kailasan Vanaja, S., Rathinam, V.A.K., Atianand, M.K., Kalantari, P., Skehan, B., Fitzgerald, K.A., and Leong, J.M. (2014). Bacterial RNA:DNA hybrids are activators of the NLRP3 inflammasome. Proc Natl Acad Sci USA 111, 7765-7770.

Khare, S., Ratsimandresy, R.A., de Almeida, L., Cuda, C.M., Rellick, S.L., Misharin, A.V., Wallin, M.C., Gangopadhyay, A., Forte, E., Gottwein, E., Perlman, H., Reed, J.C., Greaves, D.R., Dorfleutner, A., and Stehlik, C. (2014). The PYRIN domain-only protein POP3 inhibits ALR inflammasomes and regulates responses to infection with DNA viruses. Nat Immunol 15, 343-353.

Krishnaswamy, J.K., Liu, D., and Eisenbarth, S.C. (2014). POP goes the inflammasome. Nat Immunol 15, 311-313.

Leifer, C.A., and Medvedev, A.E. (2016). Molecular mechanisms of regulation of Toll-like receptor signaling. J Leukocyte Biol 100, 927-941.

Li, X., Zhang, Q., Ding, Y., Liu, Y., Zhao, D., Zhao, K., Shen, Q., Liu, X., Zhu, X., Li, N., Cheng, Z., Fan, G., Wang, Q., and Cao, X. (2016). Methyltransferase Dnmt3a upregulates HDAC9 to deacetylate the kinase TBK1 for activation of antiviral innate immunity. Nat Immunol 17, 806-815.

Liu, J., Han, C., Xie, B., Wu, Y., Liu, S., Chen, K., Xia, M., Zhang, Y.,
Song, L., Li, Z., Zhang, T., Ma, F., Wang, Q., Wang, J., Deng, K., Zhuang, Y., Wu, X., Yu, Y., Xu, T., and Cao, X. (2014). Rhbdd3 controls autoimmunity by suppressing the production of IL- 6 by dendritic cells via K27-linked ubiquitination of the regulator NEMO. Nat Immunol 15, 612-622.

Liu, J., Liu, S., Xia, M., Xu, S., Wang, C., Bao, Y., Jiang, M., Wu, Y., Xu, T., and Cao, X. (2013a). Rhomboid domain-containing protein 3 is a negative regulator of TLR3-triggered natural killer cell activation. Proc Natl Acad Sci USA 110, 7814-7819.

Liu, J., Qian, C., and Cao, X. (2016). Post-translational modification control of innate immunity. Immunity 45, 15-30.

Liu, X., Zhang, P., Bao, Y., Han, Y., Wang, Y., Zhang, Q., Zhan, Z., Meng, J., Li, Y., Li, N., Zhang, W.J., and Cao, X. (2013b). Zinc finger protein ZBTB20 promotes toll-like receptor-triggered innate immune responses by repressing I $\kappa \mathrm{B} \alpha$ gene transcription. Proc Natl Acad Sci USA 110, $11097-11102$.

Liu, Y., Zhang, Q., Ding, Y., Li, X., Zhao, D., Zhao, K., Guo, Z., and Cao, X. (2015). Histone lysine methyltransferase Ezh1 promotes TLR-triggered inflammatory cytokine production by suppressing Tollip. J Immunol 194, 2838-2846.

Long, L., Deng, Y., Yao, F., Guan, D., Feng, Y., Jiang, H., Li, X., Hu, P., Lu, X., Wang, H., Li, J., Gao, X., and Xie, D. (2014). Recruitment of phosphatase PP2A by RACK1 adaptor protein deactivates transcription factor IRF3 and limits type I interferon signaling. Immunity 40, 515-529.

Luo, W.W., Li, S., Li, C., Lian, H., Yang, Q., Zhong, B., and Shu, H.B. (2016). iRhom 2 is essential for innate immunity to DNA viruses by mediating trafficking and stability of the adaptor STING. Nat Immunol 17, 1057-1066.

Man, S.M., Karki, R., Malireddi, R.K.S., Neale, G., Vogel, P., Yamamoto, M., Lamkanfi, M., and Kanneganti, T.D. (2015). The transcription factor IRF1 and guanylate-binding proteins target activation of the AIM2 inflammasome by Francisella infection. Nat Immunol 16, 467-475.

Meunier, E., Wallet, P., Dreier, R.F., Costanzo, S., Anton, L., Rühl, S., Dussurgey, S., Dick, M.S., Kistner, A., Rigard, M., Degrandi, D., Pfeffer, K., Yamamoto, M., Henry, T., and Broz, P. (2015). Guanylate-binding proteins promote activation of the AIM2 inflammasome during infection with Francisella novicida. Nat Immunol 16, 476-484.

Nair-Gupta, P., Baccarini, A., Tung, N., Seyffer, F., Florey, O., Huang, Y., Banerjee, M., Overholtzer, M., Roche, P.A., Tampé, R., Brown, B.D., Amsen, D., Whiteheart, S.W., and Blander, J.M. (2014). TLR signals induce phagosomal MHC-I delivery from the endosomal recycling compartment to allow cross-presentation. Cell 158, 506-521.

Netea, M.G., Joosten, L.A.B., van der Meer, J.W.M., Kullberg, B.J., and van de Veerdonk, F.L. (2015). Immune defence against Candida fungal infections. Nat Rev Immunol 15, 630-642.

Nikolic, T., Movita, D., Lambers, M.E.H., Ribeiro de Almeida, C., Biesta, P., Kreefft, K., de Bruijn, M.J.W., Bergen, I., Galjart, N., Boonstra, A., and Hendriks, R. (2014). The DNA-binding factor Ctcf critically controls gene expression in macrophages. Cell Mol Immunol 11, 58-70.

Paludan, S.R., and Bowie, A.G. (2013). Immune sensing of DNA. Immunity 38, 870-880.

Qian, C., and Cao, X. (2013). Regulation of Toll-like receptor signaling pathways in innate immune responses. Ann NY Acad Sci 1283, 67-74.

Qian, C., Liu, J., and Cao, X. (2014). Innate signaling in the inflammatory immune disorders. Cytokine Growth Factor Rev 25, 731-738.

Qiao, Q., and Wu, H. (2015). Supramolecular organizing centers (SMOCs) as signaling machines in innate immune activation. Sci China Life Sci 58, 1067-1072.

Rajsbaum, R., Versteeg, G.A., Schmid, S., Maestre, A.M., Belicha-Villanueva, A., Martínez-Romero, C., Patel, J.R., Morrison, J., Pisanelli, G., Miorin, L., Laurent-Rolle, M., Moulton, H.M., Stein, D.A., Fernandez-Sesma, A., tenOever, B.R., and García-Sastre, A. (2014). Unanchored K48-linked polyubiquitin synthesized by the E3-ubiquitin ligase TRIM6 stimulates the interferon-IKK $\varepsilon$ kinase-mediated antiviral response. Immunity 40, 880-895.

Roers, A., Hiller, B., and Hornung, V. (2016). Recognition of endogenous 
nucleic acids by the innate immune system. Immunity 44, 739-754.

Schoggins, J.W., MacDuff, D.A., Imanaka, N., Gainey, M.D., Shrestha, B., Eitson, J.L., Mar, K.B., Richardson, R.B., Ratushny, A.V., Litvak, V., Dabelic, R., Manicassamy, B., Aitchison, J.D., Aderem, A., Elliott, R.M., García-Sastre, A., Racaniello, V., Snijder, E.J., Yokoyama, W.M., Diamond, M.S., Virgin, H.W., and Rice, C.M. (2014). Pan-viral specificity of IFN-induced genes reveals new roles for cGAS in innate immunity. Nature 505, 691-695.

Sellge, G., and Kufer, T.A. (2015). PRR-signaling pathways: learning from microbial tactics. Semin Immunol 27, 75-84.

Sorbara, M.T., Ellison, L.K., Ramjeet, M., Travassos, L.H., Jones, N.L., Girardin, S.E., and Philpott, D.J. (2013). The protein ATG16L1 suppresses inflammatory cytokines induced by the intracellular sensors Nod1 and Nod2 in an autophagy-independent manner. Immunity 39, 858-873.

Tang, S., Chen, T., Yu, Z., Zhu, X., Yang, M., Xie, B., Li, N., Cao, X., and Wang, J. (2014). RasGRP3 limits Toll-like receptor-triggered inflammatory response in macrophages by activating Rap1 small GTPase. Nat Commun 5, 4657.

Thaiss, C.A., Levy, M., Itav, S., and Elinav, E. (2016). Integration of innate immune signaling. Trends Immunol 37, 84-101.

Tiruppathi, C., Soni, D., Wang, D.M., Xue, J., Singh, V., Thippegowda, P.B., Cheppudira, B.P., Mishra, R.K., Debroy, A., Qian, Z., Bachmaier, K., Zhao, Y.Y., Christman, J.W., Vogel, S.M., Ma, A., and Malik, A.B. (2014). The transcription factor DREAM represses the deubiquitinase A20 and mediates inflammation. Nat Immunol 15, 239-247.

Vande Walle, L., Van Opdenbosch, N., Jacques, P., Fossoul, A., Verheugen, E., Vogel, P., Beyaert, R., Elewaut, D., Kanneganti, T.D., van Loo, G., and Lamkanfi, M. (2014). Negative regulation of the NLRP3 inflammasome by A20 protects against arthritis. Nature 512, 69-73.

Vladimer, G.I., GÃ³na, M.W., and Superti-Furga, G. (2014). IFITs: emerging roles as key anti-viral proteins. Front Immunol 5, 94.

Wang, J., Li, B.X., Ge, P.P., Li, J., Wang, Q., Gao, G.F., Qiu, X.B., and Liu, C.H. (2015). Mycobacterium tuberculosis suppresses innate immunity by coopting the host ubiquitin system. Nat Immunol 16, 237-245.

Wang, W., Jiang, M., Liu, S., Zhang, S., Liu, W., Ma, Y., Zhang, L., Zhang, J., and Cao, X. (2016). RNF122 suppresses antiviral type I interferon production by targeting RIG-I CARDs to mediate RIG-I degradation. Proc Natl Acad Sci USA 113, 9581-9586.

Wang, X., Jiang, W., Yan, Y., Gong, T., Han, J., Tian, Z., and Zhou, R. (2014). RNA viruses promote activation of the NLRP3 inflammasome through a RIP1-RIP3-DRP1 signaling pathway. Nat Immunol 15, 1126-1133.

Wen, H., Miao, E.A., and Ting, J.P.Y. (2013). Mechanisms of NOD-like receptor-associated inflammasome activation. Immunity 39, 432-441.

Wlodarska, M., Thaiss, C.A., Nowarski, R., Henao-Mejia, J., Zhang, J.P., Brown, E.M., Frankel, G., Levy, M., Katz, M.N., Philbrick, W.M., Elinav, E., Finlay, B.B., and Flavell, R.A. (2014). NLRP6 inflammasome orchestrates the colonic host-microbial interface by regulating goblet cell mucus secretion. Cell 156, 1045-1059.

Xia, M., Liu, J., Wu, X., Liu, S., Li, G., Han, C., Song, L., Li, Z., Wang, Q., Wang, J., Xu, T., and Cao, X. (2013). Histone methyltransferase Ash11 suppresses interleukin-6 production and inflammatory autoimmune diseases by inducing the ubiquitin-editing enzyme A20. Immunity 39, 470-481.

Xia, P., Wang, S., Ye, B., Du, Y., Huang, G., Zhu, P., and Fan, Z. (2015). Sox2 functions as a sequence-specific DNA sensor in neutrophils to initiate innate immunity against microbial infection. Nat Immunol 16, 366-375.

Xiao, Y., Liu, F., Yang, J., Zhong, M., Zhang, E., Li, Y., Zhou, D., Cao, Y., Li, W., Yu, J., Yang, Y., and Yan, H. (2015). Over-activation of TLR5 signaling by high-dose flagellin induces liver injury in mice. Cell Mol Immunol 12, 729-742.

Xu, S., Liu, X., Bao, Y., Zhu, X., Han, C., Zhang, P., Zhang, X., Li, W., and Cao, X. (2012). Constitutive MHC class I molecules negatively regulate TLR-triggered inflammatory responses via the Fps-SHP-2 pathway. Nat
Immunol 13, 551-559.

Yan, Y., Jiang, W., Liu, L., Wang, X., Ding, C., Tian, Z., and Zhou, R. (2015). Dopamine controls systemic inflammation through inhibition of NLRP3 inflammasome. Cell 160, 62-73.

Yan, Y., Jiang, W., Spinetti, T., Tardivel, A., Castillo, R., Bourquin, C., Guarda, G., Tian, Z., Tschopp, J., and Zhou, R. (2013). Omega-3 fatty acids prevent inflammation and metabolic disorder through inhibition of NLRP3 inflammasome activation. Immunity 38, 1154-1163.

Yang, J.L., Xu, H., and Shao, F. (2014). The immunological function of familial Mediterranean fever disease protein Pyrin. Sci China Life Sci $57,1156-1161$.

Yao, Z., Zhang, Q., Li, X., Zhao, D., Liu, Y., Zhao, K., Liu, Y., Wang, C., Jiang, M., Li, N., and Cao, X. (2014). Death Domain-associated protein 6 (Daxx) selectively represses IL-6 transcription through histone deacetylase 1 (HDAC1)-mediated histone deacetylation in macrophages. J Biol Chem 289, 9372-9379.

Yoneyama, M., Onomoto, K., Jogi, M., Akaboshi, T., and Fujita, T. (2015). Viral RNA detection by RIG-I-like receptors. Curr Opin Immunol 32, $48-53$

Yuan, R., and Li, L. (2016). Dynamic modulation of innate immunity programming and memory. Sci China Life Sci 59, 38-43.

Zhang, J., Hu, M.M., Shu, H.B., and Li, S. (2014a). Death-associated protein kinase 1 is an IRF3/7-interacting protein that is involved in the cellular antiviral immune response. Cell Mol Immunol 11, 245-252.

Zhang, L., Mo, J., Swanson, K.V., Wen, H., Petrucelli, A., Gregory, S.M., Zhang, Z., Schneider, M., Jiang, Y., Fitzgerald, K.A., Ouyang, S., Liu, Z.J., Damania, B., Shu, H.B., Duncan, J.A., and Ting, J.P.Y. (2014b). NLRC3, a member of the NLR family of proteins, is a negative regulator of innate immune signaling induced by the DNA sensor STING. Immunity 40, 329-341.

Zhang, Q., Zhao, K., Shen, Q., Han, Y., Gu, Y., Li, X., Zhao, D., Liu, Y., Wang, C., Zhang, X., Su, X., Liu, J., Ge, W., Levine, R.L., Li, N., and Cao, X. (2015). Tet2 is required to resolve inflammation by recruiting Hdac2 to specifically repress IL-6. Nature 525, 389-393.

Zhang, Y., and Li, Y.K. (2013). Regulation of innate receptor pathways by microRNAs. Sci China Life Sci 56, 13-18.

Zhang, Y., Lu, Y., Ma, L., Cao, X., Xiao, J., Chen, J., Jiao, S., Gao, Y., Liu, C., Duan, Z., Li, D., He, Y., Wei, B., and Wang, H. (2014c). Activation of vascular endothelial growth factor receptor-3 in macrophages restrains TLR4-NF- $\mathrm{KB}$ signaling and protects against endotoxin shock. Immun 40, 501-514

Zhang, Z., Bao, M., Lu, N., Weng, L., Yuan, B., and Liu, Y.J. (2013). The E3 ubiquitin ligase TRIM21 negatively regulates the innate immune response to intracellular double-stranded DNA. Nat Immunol 14, 172-178.

Zhao, K., Zhang, Q., Li, X., Zhao, D., Liu, Y., Shen, Q., Yang, M., Wang, C., Li, N., and Cao, X. (2016). Cytoplasmic STAT4 promotes antiviral type I IFN production by blocking CHIP-mediated degradation of RIG-I. J Immunol 196, 1209-1217.

Zhao, Y., and Shao, F. (2016). Diverse mechanisms for inflammasome sensing of cytosolic bacteria and bacterial virulence. Curr Opin Microbiol $29,37-42$.

Zheng, Q., Hou, J., Zhou, Y., Yang, Y., Xie, B., and Cao, X. (2015). Siglec1 suppresses antiviral innate immune response by inducing TBK1 degradation via the ubiquitin ligase TRIM27. Cell Res 25, 1121-1136.

Zhu, J., Zhang, Y., Ghosh, A., Cuevas, R.A., Forero, A., Dhar, J., Ibsen, M.S., Schmid-Burgk, J.L., Schmidt, T., Ganapathiraju, M.K., Fujita, T., Hartmann, R., Barik, S., Hornung, V., Coyne, C.B., and Sarkar, S.N. (2014). Antiviral activity of human OASL protein is mediated by enhancing signaling of the RIG-I RNA sensor. Immunity 40, 936-948.

Zhu, L.L., Zhao, X.Q., Jiang, C., You, Y., Chen, X.P., Jiang, Y.Y., Jia, X.M., and Lin, X. (2013). C-type lectin receptors dectin-3 and dectin-2 form a heterodimeric pattern-recognition receptor for host defense against fungal infection. Immunity 39, 324-334.

Open Access This article is distributed under the terms of the Creative Commons Attribution License which permits any use, distribution, and reproduction in any medium, provided the original author(s) and source are credited. 